TRANSACTIONS OF THE

AMERICAN MATHEMATICAL SOCIETY

Volume 349, Number 12, December 1997, Pages 4931-4951

S $0002-9947(97) 01871-0$

\title{
ON THE RATIONAL HOMOTOPY TYPE OF FUNCTION SPACES
}

\author{
EDGAR H. BROWN, JR. AND ROBERT H. SZCZARBA
}

\begin{abstract}
The main result of this paper is the construction of a minimal model for the function space $\mathcal{F}(X, Y)$ of continuous functions from a finite type, finite dimensional space $X$ to a finite type, nilpotent space $Y$ in terms of minimal models for $X$ and $Y$. For the component containing the constant map, $\pi_{*}(\mathcal{F}(X, Y)) \otimes Q=\pi_{*}(Y) \otimes H^{-*}(X ; Q)$ in positive dimensions. When $X$ is formal, there is a simple formula for the differential of the minimal model in terms of the differential of the minimal model for $Y$ and the coproduct of $H_{*}(X ; Q)$. We also give a version of the main result for the space of cross sections of a fibration.
\end{abstract}

\section{INTRODUCTION}

In this paper we construct a minimal model in the sense of Sullivan $[\mathrm{S}]$ for $\mathcal{F}(X, Y)$, the space of continuous functions from a space $X$ to a nilpotent space $Y$, in terms of models for $X$ and $Y$. We also generalize this to the space of sections of a bundle. We first present some of the required background material and state the main results of the paper.

Let $Y$ be a connected, nilpotent $C W$ complex (or simplicial set). A $Q$ localization of $Y$ consists of a space (or simplicial set) $Y_{Q}$ and a mapping $f: Y \rightarrow Y_{Q}$ such that $\pi_{j}\left(Y_{Q}\right)$ is a uniquely divisible group for all $j>0$ and $f_{*}: H_{j}(Y ; Q) \rightarrow H_{j}\left(Y_{Q} ; Q\right)$ is an isomorphism for $j>0$. (See [Hi].)

Let $p: E \rightarrow X$ be a nilpotent fibration with connected fibre. Recall that a $Q$ localization of $p$ consists of a fibration $\bar{p}: \bar{E} \rightarrow X$ and a fibre preserving map $f: E \rightarrow \bar{E}$ covering the identity map on $X$ and defining a $Q$ localization on the fibres.

For any graded vector space $V$, we denote by $Q[V]$ the free commutative (in the graded sense) differential graded algebra generated by $V$. If $v_{1}, \ldots, v_{n}$ is a basis for $V$, we write $Q\left[v_{1}, \ldots, v_{n}\right]$ for $Q[V]$. A commutative differential graded algebra is said to be free, nilpotent, and of finite type (FNF) if, as an algebra, $A \simeq Q[V]$, where $V=\left\{V^{q}\right\}$, the dimension of $V^{q}$ is finite and $V$ has a basis $v_{1}, v_{2}, \ldots$ such that $d v_{n+1} \in Q\left[v_{1}, \ldots, v_{n}\right]$ for all $n$. We say that $A$ is minimal if $A$ is FNF, $d v$ is decomposable for all $v \in V$, and $V^{q}=0$ for $q \leq 0$.

All algebras considered in this paper will be commutative in the graded sense, will have a unit 1 , and all algebra mappings between algebras will preserve the unit. We will consider $Q$ as a subalgebra of any algebra $A$ under the identification $r \mapsto r \cdot 1$.

Received by the editors February 12, 1996.

1991 Mathematics Subject Classification. Primary 55P15, 55P62.

(C) 1997 American Mathematical Society 
In [S], Sullivan associates to each CW complex $X$ a "rational de Rham complex" $(\Omega(X), d)$ whose homology is isomorphic to the singular cohomology of $X$ with coefficients in $Q$. He also associates to each nilpotent finite type CW complex $Y$ a minimal algebra $A$ and a homology isomorphism $\alpha: A \rightarrow \Omega(Y)$. The algebra $A$ determines the rational homotopy type of $Y$, is unique up to isomorphism, and

$$
\pi^{n}(X)=\operatorname{Hom}\left(\pi_{n}(X) \otimes Q, Q\right),
$$

is isomorphic to the space of indecomposables in $A^{n}$. (For a nilpotent group $G$, we define $G \otimes Q$ to be the direct sum $\sum\left(\Gamma_{i} / \Gamma_{i+1}\right)$, where $\left\{\Gamma_{i}\right\}$ is the lower central series of $G$.) Thus, $A=Q[V]$ with $V \simeq \pi^{*}(Y)$. The minimal algebra $A$ is called the minimal model of $X$.

Before proceeding with the statements of our results, we describe an algebraic construction which will be useful in what follows. Let $A=Q[V]$ be an FNF algebra, $B$ a $D G$ algebra such that $B^{q}$ is finite dimensional for all $q$ and $B^{q}=0$ for $q<0$. Let $B_{*}$ be the graded coalgebra with

$$
B_{q}=\operatorname{Hom}\left(B^{-q}, Q\right) \text {. }
$$

The differential on $B_{*}$ is the adjoint of the differential $d$ on $B$, and the coproduct $D: B_{*} \rightarrow B_{*} \otimes B_{*}$ is the adjoint of multiplication. Let $Q\left[A \otimes B_{*}\right]$ be the free $D G$ algebra generated by the graded vector space $A \otimes B_{*}$ with differential $d$ induced by the tensor product differential on $A \otimes B_{*}$ and let $I$ be the ideal in $Q\left[A \otimes B_{*}\right]$ generated by $1 \otimes 1-1$ and by all elements of the form

$$
a_{1} a_{2} \otimes \beta-\sum(-1)^{\left|a_{2}\right| \cdot\left|\beta_{j}^{\prime}\right|}\left(a_{1} \otimes \beta_{j}^{\prime}\right)\left(a_{2} \otimes \beta_{j}^{\prime \prime}\right),
$$

$a_{1}, a_{2} \in A, \beta \in B$, and

$$
D \beta=\sum \beta_{j}^{\prime} \otimes \beta_{j}^{\prime \prime}
$$

Then $d I \subset I$ (see Theorem 3.3) so that the differential $d$ on $Q\left[A \otimes B_{*}\right]$ defines a differential $d$ on $Q\left[A \otimes B_{*}\right] / I$.

Let $Q\left[V \otimes B_{*}\right]$ be the subalgebra of $Q\left[A \otimes B_{*}\right]$ defined by the inclusion $V \subset A=$ $Q[V]$ and let $\rho$ be the composite

$$
\rho: Q\left[V \otimes B_{*}\right] \subset Q\left[A \otimes B_{*}\right] \rightarrow Q\left[A \otimes B_{*}\right] / I .
$$

Theorem 1.2. The mapping $\rho: Q\left[V \otimes B_{*}\right] \rightarrow Q\left[A \otimes B_{*}\right] / I$ is an isomorphism of graded algebras.

This is proved in Section 3 as part of Theorem 3.5. (See also the remarks following Corollary 3.4.)

Let $d_{1}$ be the differential on $Q\left[V \otimes B_{*}\right]$ given by $d_{1}=\rho^{-1} d \rho$. By definition, $d_{1}(v \otimes \beta)$ can be computed by considering $v \otimes \beta$ as an element of $Q\left[A \otimes B_{*}\right]$, computing $d(v \otimes \beta)$ in $Q\left[A \otimes B_{*}\right]$,

$$
d(v \otimes \beta)=(d v) \otimes \beta+(-1)^{|v|} v \otimes d \beta,
$$

and then using the relations (1.1) to express $(d v) \otimes \beta$ as an element of $Q\left[V \otimes B_{*}\right]$. For example, if $d v=v_{1} v_{2}, v_{1}, v_{2} \in V$, then

$$
\begin{aligned}
(d v) \otimes \beta & =\left(v_{1} v_{2}\right) \otimes \beta \\
& =\sum(-1)^{\left|v_{2}\right| \cdot\left|\beta_{j}^{\prime}\right|}\left(v_{1} \otimes \beta_{j}^{\prime}\right)\left(v_{2} \otimes \beta_{j}^{\prime \prime}\right),
\end{aligned}
$$


where $D \beta=\sum \beta_{j}^{\prime} \otimes \beta_{j}^{\prime \prime}$. In particular, we see that the differential $d_{1}$ depends on the differentials in $A$ and $B_{*}$ and the coalgebra structure in $B_{*}$ (or equivalently, the algebra structure in $B$ ).

For any algebra $E, \Delta(E)$ will denote the rational simplicial form of $E$. (See Section 2 below or [S].) The following is a version of a result due to Haefliger, see $[\mathrm{H}]$ and $[\mathrm{V} 1]$.

Theorem 1.3. Let $X$ and $Y$ be $C W$ complexes with $Y$ nilpotent and of finite type. Let $A$ and $B$ be $D G$ algebras as above with $\alpha: A \rightarrow \Omega(Y)$ a minimal model for $Y, A=Q[V]$, and $\beta: B \rightarrow \Omega(X)$ a homology isomorphism. Then the space $\left|\Delta\left(Q\left[A \otimes B_{*}\right] / I, d\right)\right|$ and hence $\left|\Delta\left(Q\left[V \otimes B_{*}\right], d_{1}\right)\right|$ is homotopy equivalent to the function space $\mathcal{F}\left(X, Y_{Q}\right)$.

The proof is given in Sections 2 and 3. The construction $\left(Q\left[A \otimes B_{*}\right] / I, d\right)$ is the Lannes construction $(A: B)$ (see $[\mathrm{L}])$ in the category of differential graded commutative algebras. (See the remarks following Corollary 3.4.) The $(A: B)$ functor was used in [BPS] to prove a version of Theorem 1.3 with a more complex description of $d_{1}$.

The following is a stronger version of Theorem 1.3 which is proved in Section 5 .

Theorem 1.4. Let $X, Y, A$, and $B$ be as in Theorem 1.3. Then there is a differential $d_{2}$ on $Q\left[V \otimes H_{*}\left(B_{*}\right)\right]$ such that $\left|\Delta\left(Q\left[V \otimes H_{*}\left(B_{*}\right)\right], d_{2}\right)\right|$ is homotopy equivalent to $\mathcal{F}\left(X, Y_{Q}\right)$.

We note that the $Q$ localization of a component of $\mathcal{F}(X, Y)$ occurs as a component of $\mathcal{F}\left(X, Y_{Q}\right)([\mathrm{Hi}]$, Theorem 3.11).

We next state an analogue of Theorem 1.4 for the components of $\mathcal{F}\left(X, Y_{Q}\right)$.

Theorem 1.5. Let $Y$ be a nilpotent space of finite type and let $X$ be a space of finite type with $H^{q}(X ; Q)=0$ for $q>$ some $N$. For $f: X \rightarrow Y$, let $\mathcal{F}(X, Y, f)$ be the component of the function space $\mathcal{F}(X, Y)$ containing $f$. Then $\mathcal{F}(X, Y, f)$ is nilpotent and of finite type, and has a minimal model $(Q[W], d)$, where

$$
W^{q}=\left(\sum_{n} \pi^{n}(Y) \otimes H_{n-q}(X ; Q)\right) / K^{q}
$$

for subspaces $K^{q}, q>0$. If $f$ is the constant map, then $K^{q}=0$.

Corollary 1.6. The homotopy group $\pi_{q}(\mathcal{F}(X, Y, f)) \otimes Q, q>0$, is isomorphic to

$$
\left(\sum_{n}\left(\pi^{n}(Y) \otimes Q\right) \otimes H_{n-q}(X ; Q)\right) / K_{q} .
$$

If $f$ is the constant map, $K_{q}=0$.

This result was proved by Vigué-Poirrier in [V2] when $H^{p}(X ; Q)=0, p>k$, and $Y$ is $(k+1)$ connected. In Section 7 we show by an example that the homotopy groups $\pi_{q}(\mathcal{F}(X, Y, f)) \otimes Q$ can vary with $f$. Another example was given by G.W. Whitehead in the appendix of [W].

The results stated above have analogues for the space of sections in a nilpotent fibre space. For example, we have the following.

Theorem 1.7. Let $p: E \rightarrow X$ be a nilpotent finite type fibration with fibre $Y$ and $H_{q}(X ; Q)=0$ for $q>$ some $N$. Let $f: X \rightarrow E$ be a section and denote by 
$\Gamma(p, f)$ the component of the space of sections $\Gamma(p)$ containing $f$. Then $\Gamma(p, f)$ has a minimal model $A \simeq Q[W]$ where $W^{q}, q>0$, is isomorphic to a quotient of

$$
\sum_{n} \pi^{n}(Y) \otimes H_{n-q}(X ; Q) .
$$

Corollary 1.8. The homotopy group $\pi_{q}(\Gamma(p, f)) \otimes Q$ is isomorphic to a quotient of

$$
\sum_{n}\left(\pi_{n}(Y) \otimes Q\right) \otimes H_{n-q}(X ; Q) .
$$

The proofs of the results for the space of sections are straightforward extensions of those for function spaces. An outline is given in Section 4. In particular, a model for $E \rightarrow X$ takes the form $B[V]=B \otimes Q[V]$, which replaces $A$ in the above, and the ideal $I$ is modified by adding the relations $b \otimes \beta-(-1)^{\alpha(|b|)} \beta(b), b \in B, \beta \in B_{*}$.

The computation of the differential in $Q\left[V \otimes H_{*}\left(B_{*}\right)\right]$ is in general quite complicated. Examples are given in Section 7 to illustrate this. However, if $X$ is formal [S] (that is, when the minimal model for $\left(H^{*}(X, Q), d=0\right)$ is a minimal model for $X)$, the computation is much simpler, as we describe below..

Suppose that $X$ and $Y$ are as in Theorem 1.5 and that $X$ is formal with $H_{q}(X ; Q)=0$ for $q>$ some $N$. Let $B=H^{*}(X ; Q)$ be the rational cohomology ring of $X$ as a $D G$ algebra with zero differential. Thus $B_{*}=H_{*}(X ; Q)$ is the rational homology coalgebra of $X$. Let $D_{*}$ be the coproduct in $H_{*}(X, Q)$,

$$
D_{*}: H_{*}(X ; Q) \rightarrow H_{*}(X ; Q) \otimes H_{*}(X ; Q) .
$$

Applying the algebraic construction described above, we obtain a $D G$ algebra $\left(Q\left[V \otimes H_{*}(X ; Q)\right], d_{1}\right)$, where $V \simeq \pi^{*}(Y)$ and $d_{1}=\rho^{-1} d \rho$ with $\rho$ the mapping of Theorem 1.2. At the end of Section 5 we prove

Theorem 1.9. Let $X$ and $Y$ be $C W$ complexes with $Y$ nilpotent and of finite type, $X$ formal, and $H_{q}(X ; Q)=0$ for $q>$ some $N$. Then the space

$$
\left|\Delta\left(Q\left[\pi^{*}(Y) \otimes H_{*}(X ; Q)\right], d_{1}\right)\right|
$$

is homotopy equivalent to $\mathcal{F}\left(X, Y_{Q}\right)$. Furthermore, given $f: X \rightarrow Y$, there is an ideal $K$ (see Section 6) depending on $f$, closed under $d_{1}$, and such that $Q\left[V \otimes H_{*}(X ; Q)\right] / K$ is $F N F$ on positive dimensional generators and $\mathcal{F}(X, Y, f)_{Q}$ is homotopy equivalent to $\left|\Delta\left(Q\left[V \otimes H_{*}(X ; Q)\right] / K, d_{2}\right)\right|$.

For example, if $c \in H_{*}(X ; Q)$ and $v, v_{1}, v_{2} \in V$ with $d v=v_{1} v_{2}$, then

$$
d_{1}(v \otimes c)=\sum(-1)^{\left|v_{2}\right| \cdot\left|c_{j}\right|}\left(v_{1} \otimes c_{j}^{\prime}\right)\left(v_{1} \otimes c_{j}^{\prime \prime}\right),
$$

where $D_{*} c=\sum c_{j}^{\prime} \otimes c_{j}^{\prime \prime}$. Note that, in this case, $d_{1}$ depends on the differential in $A$ and the coproduct in $H_{*}(X ; Q)$.

The proof of Theorem 1.9 is given at the end of Section 5. The special case of Theorem 1.9 when $X=S^{1}$ was proved by Sullivan in [VS].

Remark 1.10. All of the above can be reformulated in terms of simplicial sets instead of CW complexes. Then all the theorems remain true with only minor modifications. In fact, step one in our proofs is to convert to simplicial sets.

Remark 1.11. In [BZ], we showed how to formulate real homotopy theory for simplicial spaces in a way which is analogous to rational homotopy theory for simplicial sets. All of the above can be converted to real homotopy theory and the theorems 
remain true if, for example, one starts with $X=\Delta(B)$ and $Y=\Delta(A)$ where $A$ and $B$ are minimal algebras over the reals instead of the rationals.

Throughout this paper, the set of morphisms between objects $S$ and $T$ in a specified category will be denoted by $(S, T)$, possibly with subscripts. For example, if $S$ and $T$ are objects in the category of differential graded algebras, then $(S, T)$ will denote the set of $D G$ algebra mappings and $(S, T)_{D G}$ the set of mappings preserving the grading and commuting with the differentials, but not necessarily preserving multiplication.

The paper is organized as follows. The proofs of the main results for function spaces are given in Sections 2, 3, and 5. In Section 4, we outline the changes necessary to extend our results on function spaces to the space of sections in a nilpotent fibration. Section 6 deals with components and Section 7 contains two examples. The first example shows that not all components of a function space have the same $Q$-homotopy type. The second example shows that the formality condition in Theorem 1.9 is necessary.

\section{An Algebraic Model for $\mathcal{F}(X, Y)_{Q}$}

For topological spaces $U$ and $W$, let $\mathcal{F}(U, W)$ be the space of all continuous functions from $U$ to $W$ in the compact open topology. The goal of this section is to reduce the problem of determining the rational homotopy type of $\mathcal{F}(U, V)$ to the determination of the homotopy type of the rational simplicial form $\Delta\left(Q\left[V \otimes B_{*}\right]\right)$ of the differential graded algebra $Q\left[V \otimes B_{*}\right]$ described in Section 1. Before proceeding, we recall some of the notions from rational homotopy theory that will be needed in what follows.

Let $\Delta \mathcal{S}$ denote the category of simplicial sets. (See $[\mathrm{M}]$ for a detailed account of homotopy theory in $\Delta \mathcal{S}$.) Recall that, for $X, Y \in \Delta \mathcal{S}$, the function space $\mathcal{F}(X, Y) \in$ $\Delta \mathcal{S}$ is defined by

$$
\mathcal{F}(X, Y)_{q}=(X \times \Delta[q], Y),
$$

where $\Delta[q]$ is the simplicial set analogue of $\Delta^{q}$ with

$$
\Delta[q]_{p}=\left\{\left(i_{0}, i_{1}, \ldots, i_{p}\right): 0 \leq i_{0} \leq i_{1} \leq \ldots \leq i_{p} \leq q\right\}
$$

and $(X \times \Delta[q], Y)$ is the set of simplicial mappings from $X \times \Delta[q]$ to $Y$. The face and degeneracy operations in $\mathcal{F}(X, Y)$ are defined in terms of the face inclusions $\Delta[q-1] \rightarrow \Delta[q]$ and degeneracy projections $\Delta[q+1] \rightarrow \Delta[q]$. (See $[\mathrm{M}]$, p. 16, for details.) For any $X \in \Delta \mathcal{S}$ and $y \in X_{0}$ let $X_{y}$ denote the simplicial subset of $X$ consisting of all simplices whose vertices are all at $y$. When $X$ is Kan, $X_{y}$ is homotopy equivalent to the component of $X$ containing $y$. For $u \in \mathcal{F}(X, Y)_{0}=$ $(X, Y)$, let $\mathcal{F}(X, Y, u)=\mathcal{F}(X, Y)_{u}$.

Let $\mathcal{A}$ be the category of differential graded commutative algebras, and let $\Omega_{q}^{p}=$ $\Omega^{p}\left(\Delta^{q}\right)$ be the vector space of rational differential $p$-forms $w$ on $\Delta^{q}$ :

$$
w=\sum a_{i_{1} \ldots i_{p}} d t_{i_{1}} \ldots d t_{i_{p}},
$$

where the $a_{i_{1} \ldots i_{p}}$ are polynomials in $t_{0}, \ldots, t_{q}$ with rational coefficients. Then $\Omega^{p}=$ $\left\{\Omega_{q}^{p}, q \geq 0\right\}$ is a simplicial set, $\Omega_{q}=\left\{\Omega_{q}^{p}, p \geq 0\right\}$ is a $D G$ algebra, and $\Omega=$ $\left\{\Omega_{q}^{p}, p, q \geq 0\right\}$ is a simplicial differential graded algebra. Define functors

$$
\Delta: \mathcal{A} \rightarrow \Delta \mathcal{S}, \quad \Omega: \Delta \mathcal{S} \rightarrow \mathcal{A}
$$


by $\Delta(A)=(A, \Omega)$ and $\Omega(X)=(X, \Omega)$. Then $\Delta(A)$ is the rational simplicial form of $A$ and $\Omega(X)$ the algebra of rational differential forms on $X$ [BG].

For $A, B \in \mathcal{A}$, let $\mathcal{F}(A, B) \in \Delta \mathcal{S}$ be the function space defined by

$$
\mathcal{F}(A, B)_{q}=\left(A, \Omega_{q} \otimes B\right),
$$

the space of all $D G$ algebra mappings from $A$ to $\Omega_{q} \otimes B$. The simplicial structure on $\mathcal{F}(A, B)$ is defined using the simplicial structure of $\Omega$. For $w \in \mathcal{F}(A, B)_{0}=(A, B)$, let $\mathcal{F}(A, B, w)=\mathcal{F}(A, B)(w)$.

We note that, in what follows, the function spaces $\mathcal{F}(X, Y)$ and $\mathcal{F}(A, B)$ will be Kan [BZ], so that $\mathcal{F}(X, Y, u)$ and $\mathcal{F}(A, B, w)$ are actually homotopy equivalent to the corresponding path components.

Our first result relates the function spaces for topological spaces and simplicial sets. Let $\Delta(U)$ denote the singular complex of the space $U$ and let $|X|$ denote the geometric realization of the simplicial set $X$.

Theorem 2.1. For $X, Y \in \Delta \mathcal{S}$, the simplicial set $\mathcal{F}(X, Y)$ has the same homotopy type as $\Delta \mathcal{F}(|X|,|Y|)$.

Proof. For $f \in \mathcal{F}(X, Y)_{q}, f: X \times \Delta[q] \rightarrow Y$, define mappings

$$
\mathcal{F}(X, Y) \stackrel{\alpha}{\longrightarrow} \Delta \mathcal{F}(|X|,|Y|) \stackrel{\beta}{\rightarrow} \mathcal{F}(X, \Delta|Y|) \stackrel{\gamma}{\rightarrow} \Delta \mathcal{F}(|X|,|(\Delta|Y|)|)
$$

in $\Delta \mathcal{S}$ as follows:

$$
\alpha(f)=|f|:|X \times \Delta[q]|=|X| \times \Delta^{q} \rightarrow|Y|,
$$

where $|\Delta[q]|$ is identified with $\Delta^{q}$.

For $g \in \Delta \mathcal{F}(|X|,|Y|)_{q}, g:|X| \times \Delta^{q} \rightarrow|Y|, \beta(g)$ is the composite

$$
\beta(g): X \times \Delta[q] \stackrel{\sigma}{\longrightarrow} \Delta|X \times \Delta[q]|=\Delta\left(|X| \times \Delta^{q}\right) \stackrel{\Delta g}{\longrightarrow} \Delta|Y|,
$$

where $\sigma: X \times \Delta[q] \rightarrow \Delta|X \times \Delta[q]|$ is the canonical inclusion.

For $h \in \mathcal{F}(X, \Delta|Y|)_{q}, h: X \times \Delta[q] \rightarrow \Delta|Y|$,

$$
\gamma(h)=|h|:|X| \times \Delta^{q} \rightarrow|(\Delta|Y|)| .
$$

It is now easily checked that the composite $\beta \alpha$ is induced by the canonical homotopy equivalence $Y \rightarrow \Delta|Y|$ and that the composite $\gamma \beta$ is induced by the canonical homotopy equivalence $|Y| \rightarrow|(\Delta|Y|)|$. It follows that

$$
\alpha_{*}: \pi_{q}(\mathcal{F}(X, Y)) \rightarrow \pi_{q}(\Delta \mathcal{F}(|X|,|Y|))
$$

is injective for all $q$. To see that $\alpha_{*}$ is surjective, consider $y \in \pi_{q}(\Delta \mathcal{F}(|X|,|Y|))$ and let $x=(\beta \alpha)_{*}^{-1} \beta_{*}(y)$. Then

$$
\begin{aligned}
(\gamma \beta)_{*}\left(\alpha_{*}(x)\right) & =\left(\gamma_{*} \beta_{*}\right) \alpha_{*}(\beta \alpha)_{*}^{-1} \beta_{*}(y) \\
& =\gamma_{*} \beta_{*}(y),
\end{aligned}
$$

so that $\alpha_{*}(x)=y$ since $(\gamma \beta)_{*}$ is an isomorphism. It follows that $\alpha$ is a homotopy equivalence.

We note that if $X=\Delta U, Y=\Delta W$ for topological spaces $U, W$, then

$$
\begin{aligned}
F(U, W) & \simeq F(|\Delta U|,|\Delta W|) \\
& \simeq|\Delta F(|\Delta U|,|\Delta W|)| \stackrel{|\alpha|}{\simeq}|F(\Delta U, \Delta W)| .
\end{aligned}
$$

As a consequence of Theorem 2.1, we can and will work in the category of simplicial sets for the remainder of the paper. 
Let $X, Y \in \Delta \mathcal{S}, Y$ connected, Kan, nilpotent of finite type and $H_{q}(X ; Q)$ finitely generated and zero for $q>$ some $N$. Suppose $u \in \mathcal{F}(X, Y)_{0}$ and $h: Y \rightarrow Y_{Q}$ is a $Q$ localization.

Theorem 2.2. The mapping $h_{*}: \mathcal{F}(X, Y, u) \rightarrow \mathcal{F}\left(X, Y_{Q}, h u\right)$ is a $Q$ localization.

Proof. See [Hi], Theorem 3.11.

Let $A, B \in \mathcal{A}, \alpha: A \rightarrow \Omega Y$ a minimal model for $Y$ and $\beta: B \rightarrow \Omega X$ a homology isomorphism. Assume further that $B^{q}$ is finite dimensional for all $q$. Then

$$
h: Y \stackrel{j}{\rightarrow} \Delta \Omega Y \stackrel{\Delta \alpha}{\longrightarrow} \Delta A
$$

is a $Q$ localization, where $j: Y \rightarrow \Delta \Omega Y$ is the canonical mapping and $\Delta \alpha: \Delta \Omega Y \rightarrow$ $\Delta A$ is induced by $\alpha$ [BZ]. Thus, $h_{*}: \mathcal{F}(X, Y, u) \rightarrow \mathcal{F}(X, \Delta A, h u)$ is a $Q$ localization. Now, according to Theorem 2.20 of [BZ], there is a homotopy equivalence

$$
\gamma: \mathcal{F}(X, \Delta A) \rightarrow \mathcal{F}(A, \Omega X)
$$

Furthermore, according to this same theorem, the mapping

$$
\beta_{*}: \mathcal{F}(A, \Omega X) \rightarrow \mathcal{F}(A, B)
$$

is also a homotopy equivalence since $\beta$ is a homology equivalence. Thus we have

Theorem 2.3. Let $\tilde{u}=\beta_{*} \gamma(h u)$. Then $\mathcal{F}(X, Y, u) \rightarrow \mathcal{F}(A, B, \tilde{u})$, is a $Q$ localization.

\section{An Algebraic Reduction}

We now proceed to analyze $\mathcal{F}(A, B)$ as described in Section 2. Let $B_{*}$ be the differential, graded coalgebra with coproduct $D$ and grading

$$
B_{q}=\operatorname{Hom}\left(B^{-q}, Q\right),
$$

$q \geq 0$, with differential the dual of the differential $d$ on $B$. (We will also use $d$ to denote the differential on $A$ and on $B_{*}$, since no confusion seems likely.) Let $\left\{b_{i}\right\}$ be a basis for $B$ and $\left\{\beta_{i}\right\}$ the dual basis for $B_{*}$. For $b \in B$, let $|b|$ denote the degree of $b$ and for any integer $n$, let $\alpha(n)=[(n+1) / 2]$, the greatest integer in $(n+1) / 2$. Define

$$
\Psi:\left(A \otimes B_{*}, \Omega\right)_{D G} \rightarrow(A, \Omega \otimes B)_{D G}
$$

in $\Delta \mathcal{S}$ by

$$
\Psi(\omega)(a)=\sum_{i}(-1)^{\alpha\left(\left|b_{i}\right|\right)} \omega\left(a \otimes \beta_{i}\right) \otimes b_{i}
$$

Note that the sum is finite since $\omega\left(a \otimes \beta_{i}\right)=0$ whenever degree $\left(a \otimes \beta_{i}\right)<0$, since $\Omega$ is nonnegatively graded and $B^{q}$ is finite dimensional.

Theorem 3.1. The mapping $\Psi$ is a simplicial isomorphism.

Proof. Before proving this result, we define two useful mappings. Let

$$
\varepsilon: Q \rightarrow B_{*} \otimes B, \quad \eta: B \otimes B_{*} \rightarrow Q
$$

be the mappings defined by

$$
\begin{aligned}
\varepsilon(1) & =\sum_{i}(-1)^{\alpha\left(\left|b_{i}\right|\right)} \beta_{i} \otimes b_{i}, \\
\eta(b \otimes \beta) & =(-1)^{\alpha(|b|)} \beta(b) .
\end{aligned}
$$


Note that, strictly speaking, $\varepsilon(1)$ is not an element of $B \otimes B_{*}$, since the sum may be infinite. However, when $\varepsilon$ occurs in what follows, only a finite number of terms will be involved.

Lemma 3.2. The mappings $\varepsilon$ and $\eta$ above preserve grading and commute with differentials.

Here we consider $Q$ as the differential graded algebra with $Q$ in degree zero and 0 in degrees different from zero.

Proof. The first assertion is obvious. The second will be proved if we can show that $d \circ \varepsilon=\eta \circ d=0$. We begin by showing that $d \circ \varepsilon=0$.

Let $a_{i}^{j} \in Q$ be defined by

$$
d b_{i}=\sum_{j} a_{i}^{j} b_{j}
$$

so that

$$
d \beta_{i}=\sum_{j} a_{j}^{i} \beta_{j}
$$

Then

$$
\begin{aligned}
d \varepsilon(1) & =\sum_{i}(-1)^{\alpha\left(\left|b_{i}\right|\right)}\left(d^{*} \beta_{i} \otimes b_{i}+(-1)^{\left|b^{i}\right|} \beta_{i} \otimes d b_{i}\right) \\
& =\sum_{i, j}(-1)^{\alpha\left(\left|b_{i}\right|\right)} a_{j}^{i} \beta_{j} \otimes b_{i}+\sum_{i, j}(-1)^{\alpha\left(\left|b_{i}\right|\right)+\left|b^{i}\right|} a_{i}^{j} \beta_{i} \otimes b_{j} .
\end{aligned}
$$

Interchanging the roles of $i$ and $j$ in the first sum and using the fact that $a_{j}^{i}=0$ unless $\left|b_{j}\right|=\left|b_{i}\right|+1$, we have

$$
d \varepsilon(1)=\sum_{i, j}\left((-1)^{\alpha\left(\left|b_{i}\right|+1\right)}+(-1)^{\alpha\left(\left|b_{i}\right|\right)+\left|b_{i}\right|}\right) a_{i}^{j} \beta_{i} \otimes b_{j} .
$$

The fact that this expression vanishes is a consequence of the easily verified identity

$$
[(n+2) / 2]=1+[(n+1) / 2]+n \quad \bmod 2 .
$$

The equation $\eta(d(b \otimes \beta))=0$ is proved in the same way. We leave the details to the reader.

For $u \in(A, \Omega \otimes B)_{D G}$, let $\Theta(u)$ be the composite

$$
A \otimes B_{*} \stackrel{u \otimes i d}{\longrightarrow} \Omega \otimes B \otimes B_{*} \stackrel{i d \otimes \eta}{\longrightarrow} \Omega \otimes Q \simeq \Omega .
$$

Note that $\Psi(\omega)$ is the composite

$$
A \rightarrow A \otimes Q \stackrel{i d \otimes \varepsilon}{\longrightarrow} A \otimes B_{*} \otimes B \stackrel{\omega \otimes i d}{\longrightarrow} \Omega \otimes B,
$$

and that $\Theta$ can be written more explicitly as

$$
\Theta(u)\left(a \otimes \beta_{i}\right)=(-1)^{\alpha\left(\left|b_{i}\right|\right)} \omega_{i},
$$

where $u(a)=\sum_{j} \omega_{j} \otimes b_{j}$ 
Now, if $u$ and $\omega$ preserve grading and differentials, then each of the mappings in the definition of $\Theta(u)$ and $\Psi(\omega)$ also preserves grading and differentials. Thus we have simplicial mappings

$$
\begin{aligned}
\Theta:(A, \Omega \otimes B)_{G D} & \rightarrow\left(A \otimes B_{*}, \Omega\right)_{G D}, \\
\Psi:\left(A \otimes B_{*}, \Omega\right)_{G D} & \rightarrow(A, \Omega \otimes B)_{G D}
\end{aligned}
$$

which satisfy

$$
\begin{aligned}
\Psi(\Theta(u))(a) & =\sum_{i}(-1)^{\alpha\left(\left|b_{i}\right|\right)} \Theta(u)\left(a \otimes \beta_{i}\right) \otimes b_{i} \\
& =\sum_{i} \omega_{i} \otimes b_{i}=u(a)
\end{aligned}
$$

and

$$
\Theta(\Psi(\omega))\left(a \otimes \beta_{i}\right)=\omega\left(a \otimes \beta_{i}\right),
$$

since $\Psi(\omega)(a)=\sum_{i} \omega_{i} \otimes b_{i}=\sum_{i}(-1)^{\alpha\left(\left|b_{i}\right|\right)} \omega\left(a \otimes \beta_{i}\right) \otimes b_{i}$. Thus each of the composites $\Psi \circ \Theta$ and $\Theta \circ \Psi$ is the identity, and Theorem 3.1 is proved.

Consider now $Q\left[A \otimes B_{*}\right]$, the free commutative $D G$ algebra on the differential graded vector space $A \otimes B_{*}$. Each $\omega \in\left(A \otimes B_{*}, \Omega\right)_{D G}$ extends to a unique $D G$ algebra mapping $\omega: Q\left[A \otimes B_{*}\right] \rightarrow \Omega$. Thus we can identify $\left(A \otimes B_{*}, \Omega\right)_{D G}$ with $\left(Q\left[A \otimes B_{*}\right], \Omega\right)$, the $D G$ algebra mappings, and consider $\Psi$ as a simplicial isomorphism

$$
\Psi:\left(Q\left[A \otimes B_{*}\right], \Omega\right) \rightarrow(A, \Omega \otimes B)_{D G} .
$$

Let $\sigma: A \otimes A \otimes B_{*} \rightarrow Q\left[A \otimes B_{*}\right]$ be given by

$$
\sigma\left(a_{1} \otimes a_{2} \otimes \beta\right)=a_{1} a_{2} \otimes \beta-\sum_{j}(-1)^{\left|a_{2}\right| \cdot\left|\beta_{j}^{\prime}\right|}\left(a_{1} \otimes \beta_{j}^{\prime}\right)\left(a_{2} \otimes \beta_{j}^{\prime \prime}\right) .
$$

where $D \beta=\sum \beta_{j}^{\prime} \otimes \beta_{j}^{\prime \prime}$.

Theorem 3.3. For $\omega \in\left(Q\left[A \otimes B_{*}\right], \Omega\right)$, the mapping $\Psi(\omega) \in(A, \Omega \otimes B)_{D G}$ is a $D G$ algebra mapping if and only if $\omega\left(1 \otimes 1^{*}\right)=1$ and $\omega\left(\sigma\left(a_{1} \otimes a_{2} \otimes \beta_{i}\right)\right)=0$ for all $a_{1}, a_{2} \in A$ and all $\beta \in B_{*}$. Furthermore, if $I \subset Q\left[A \otimes B_{*}\right]$ is the ideal generated by $1 \otimes 1^{*}-1$ and $\sigma\left(a_{1} \otimes a_{2} \otimes b\right), a_{1}, a_{2} \in A, b \in B_{*}$, then $d I \subset I$.

Corollary 3.4. The mapping $\Psi$ defines a simiplicial isomorphism

$$
\Psi:\left(Q\left[A \otimes B_{*}\right] / I, \Omega\right) \rightarrow(A, \Omega \otimes B) .
$$

Remark. It follows that $\left(Q\left[A \otimes B_{*}\right] / I, d\right)$ is a realization of the Lannes functor $A: B$ in the category $\mathcal{A}$. One can view the Lannes machinery as providing a homological algebra type approach to the preceding algebra. In particular, Theorem 1.2 can be proved in the Lannes context using Theorem 2.1 [BPS].

Proof of Theorem 3.3. We first note that an element $z \in \Omega \otimes B$ is zero if and only if $\left(i d_{\Omega} \otimes \eta\right)\left(z \otimes \beta_{k}\right)=0$ for all $k$.

Using the fact that

$$
\alpha(n+m)=\alpha(n)+\alpha(m)+n m \bmod 2
$$

for all non-negative integers $n$ and $m$, a straightforward computation shows that

$$
\left(i d_{\Omega} \otimes \eta\right)\left(\left(\Psi(\omega)\left(a_{1} a_{2}\right)-\left(\Psi(\omega)\left(a_{1}\right)\right)\left(\Psi(\omega)\left(a_{2}\right)\right)\right) \otimes \beta_{k}\right)=\omega\left(\sigma\left(a_{1} \otimes a_{2} \otimes \beta_{k}\right)\right),
$$


which proves the first part of Theorem 3.3. To prove that $d I \subset I$, consider the mappings

$$
\begin{aligned}
& \mu: A \otimes A \rightarrow A, \\
& D: B_{*} \rightarrow B_{*} \otimes B_{*}, \\
& \bar{\mu}: Q\left[A \otimes B_{*}\right] \otimes Q\left[A \otimes B_{*}\right] \rightarrow Q\left[A \otimes B_{*}\right], \\
& \tau: A \otimes A \otimes B_{*} \otimes B_{*} \rightarrow A \otimes B_{*} \otimes A \otimes B_{*},
\end{aligned}
$$

where $\mu$ is the multiplication in $A, D$ the comultiplication in $B_{*}, \bar{\mu}$ is the multiplication in $Q\left[A \otimes B_{*}\right]$, and

$$
\tau\left(a \otimes a^{\prime} \otimes \beta \otimes \beta^{\prime}\right)=(-1)^{\left|a^{\prime}\right| \cdot|\beta|} a \otimes \beta \otimes a^{\prime} \otimes \beta^{\prime} .
$$

Then $\sigma=\mu \otimes i d_{B^{*}}-\bar{\mu} \tau\left(i d_{A \otimes A} \otimes D\right)$ and, since each of the mappings on the right commutes with the corresponding differential, so does $\sigma$, and the theorem is proved.

Let $A=Q[V]$, where $V^{q}$ is finite dimensional for all $q$, and let $\rho$ be the composite

$$
\rho: Q\left[V \otimes B_{*}\right] \subset Q\left[A \otimes B_{*}\right] \rightarrow Q\left[A \otimes B_{*}\right] / I .
$$

Theorem 3.5. The mapping $\rho: Q\left[V \otimes B_{*}\right] \rightarrow Q\left[A \otimes B_{*}\right] / I$ is an isomorphism of graded algebras.

Let $d_{1}$ be the differential on $Q\left[V \otimes B_{*}\right]$ defined by $d_{1}=\rho^{-1} d \rho$, where $d$ is the differential on $Q\left[A \otimes B_{*}\right] / I$ induced by the tensor product differential on $A \otimes B_{*}$. We then have

Corollary 3.6. Let $A=Q[V], B \in \mathcal{A}, \alpha: A \rightarrow \Omega Y$ a minimal model for $Y$, and $\beta: B \rightarrow \Omega X$ a homology isomorphism. Assume further that $B^{q}$ is finite dimensional for all $q$. Then the simplicial set $\Delta\left(Q\left[V \otimes B_{*}\right], d_{1}\right)$ is homotopy equivalent to $\mathcal{F}\left(X, Y_{Q}\right)$.

The proof of Theorem 3.5 proceeds by constructing an inverse for $\rho$. We begin with some preliminaries.

Given $v_{1}, \ldots, v_{k} \in V$ and $\beta_{\ell} \in B_{*}$, define $T\left(v_{1}, \ldots, v_{k}, \beta_{\ell}\right)$ in $Q\left[V \otimes B_{*}\right]$ inductively by

$$
\begin{aligned}
T\left(v_{1}, \ldots, v_{k}, \beta_{\ell}\right) & =v_{1} \otimes \beta_{\ell} \text { if } k=1, \\
& =\sum_{i, j} \varepsilon_{i j}^{k} \lambda_{i j}^{\ell} T\left(v_{1}, \ldots, v_{k-1}, \beta_{i}\right)\left(v_{k} \otimes \beta_{j}\right) \text { if } k>1,
\end{aligned}
$$

where

$$
\begin{aligned}
& D\left(\beta_{\ell}\right)=\sum \lambda_{i j}^{\ell} \beta_{i} \otimes \beta_{j}, \\
& \varepsilon_{i j}^{k}=(-1)^{\left|\beta_{i}\right|\left(\left|\beta_{j}\right|+\left|v_{k}\right|\right)} .
\end{aligned}
$$

Lemma 3.7. For any $v_{1}, \ldots, v_{k} \in V, \beta_{\ell} \in B_{*}, 1<m \leq k$, we have

$$
T\left(v_{1}, \ldots, v_{k}, \beta_{\ell}\right)=\sum_{i, j} \varepsilon_{i j}^{m k} \lambda_{i j}^{\ell} T\left(v_{1}, \ldots, v_{m-1}, \beta_{i}\right) T\left(v_{m}, \ldots, v_{k}, \beta_{j}\right),
$$

where

$$
\varepsilon_{i j}^{m k}=(-1)^{\left|\beta_{i}\right|\left(\left|\beta_{j}\right|+\left|v_{m}\right|+\cdots+\left|v_{k}\right|\right)} .
$$


Proof. We proceed by induction on $k$. The case $k=2$ follows immediately from the definition of $T$. Suppose the result is true for $k-1$ and let $1<m \leq k$. If $m=k$, the result again follows from the definition of $T$, so we can assume $1<m<k$. Then, by definition, $T\left(v_{1}, \ldots, v_{k}, \beta_{\ell}\right)$ is given by

$$
\begin{aligned}
& \sum_{i, j} \varepsilon_{i j}^{k} \lambda_{i j}^{\ell} T\left(v_{1}, \ldots, v_{k-1}, \beta_{i}\right)\left(v_{k} \otimes \beta_{j}\right) \\
& =\sum_{i, j} \varepsilon_{i j}^{k} \lambda_{i j}^{\ell} \sum_{r, s} \varepsilon_{r s}^{m k-1} \lambda_{r s}^{i} T\left(v_{1}, \ldots, v_{m-1}, \beta_{r}\right) T\left(v_{m}, \ldots v_{k-1}, \beta_{s}\right)\left(v_{k} \otimes \beta_{j}\right)
\end{aligned}
$$

by induction. Now $\lambda_{i j}^{\ell} \lambda_{r s}^{i}=\lambda_{s j}^{i} \lambda_{r i}^{\ell}$, since multiplication in $B$ is associative. Making this substitution and rearranging terms, we can express $T\left(v_{1}, \ldots, v_{m}, \beta_{\ell}\right)$ as

$$
\sum_{r, j} \varepsilon_{i j}^{k} \varepsilon_{r s}^{m k-1} \lambda_{r i}^{\ell} T\left(v_{1}, \ldots, v_{m-1}, \beta_{k}\right) \sum_{s, j} \lambda_{s j}^{i} T\left(v_{m}, \ldots, v_{k-1}, \beta_{s}\right)\left(v_{k} \otimes \beta_{j}\right) .
$$

Except for a factor $\varepsilon_{s j}^{k}$, the second sum on the right is $T\left(v_{m}, \ldots, v_{k}, \beta_{i}\right)$ by definition. Inserting this factor twice, we have

$$
T\left(v_{1}, \ldots, v_{k}, \beta_{\ell}\right)=\sum_{r, i} \varepsilon_{i j}^{k} \varepsilon_{r s}^{m k-1} \varepsilon_{s j}^{k} \lambda_{r i}^{\ell} T\left(v_{1}, \ldots, v_{m-1}, \beta_{k}\right) T\left(v_{m}, \ldots, v_{k}, \beta_{i}\right) .
$$

Using the fact that $\lambda_{s j}^{i}=0$ unless $\left|\beta_{s}\right|+\left|\beta_{j}\right|=\left|\beta_{i}\right|$ and $\lambda_{r i}^{\ell}=0$ unless $\left|\beta_{r}\right|+\left|\beta_{i}\right|=$ $\left|\beta_{\ell}\right|$, we see that $\left|\beta_{j}\right|=\left|\beta_{r}\right| \bmod 2$ and $\left|\beta_{s}\right|=\left|\beta_{r}\right|+\left|\beta_{i}\right| \bmod 2$. An easy computation now shows that

$$
\varepsilon_{i j}^{k} \varepsilon_{r s}^{m k-1} \varepsilon_{s j}^{k}=\varepsilon_{r i}^{k},
$$

which gives the required result

Lemma 3.8. For any $v_{1}, \ldots, v_{k} \in V, \beta_{\ell} \in B_{*}, 1 \leq m<k$, we have

$$
T\left(v_{1}, \ldots, v_{k}, \beta_{\ell}\right)=(-1)^{\left|v_{m}\right| \cdot\left|v_{m+1}\right|} T\left(v_{1}, \ldots, v_{m-1}, v_{m+1}, v_{m}, \ldots, v_{k}, \beta_{\ell}\right) .
$$

Proof. For $k=2$ we have

$$
\begin{aligned}
T\left(v_{1}, v_{2}, \beta_{\ell}\right) & =\sum_{i, j} \varepsilon_{i j}^{2} \lambda_{i j}^{\ell}\left(v_{1} \otimes \beta_{i}\right)\left(v_{2} \otimes \beta_{j}\right) \\
& =\sum_{i, j} \varepsilon_{i j}^{2}(-1)^{\left(\left|v_{1}\right|+\left|b^{i}\right|\right)\left(\left|v_{2}\right|+\left|\beta_{j}\right|\right)+\left|\beta_{i}\right| \cdot\left|b^{j}\right|} \lambda_{j i}^{\ell}\left(v_{2} \otimes \beta_{j}\right)\left(v_{1} \otimes \beta_{i}\right) \\
& =\sum \varepsilon_{j i}^{1}(-1)^{\left|v_{1}\right|\left|v_{2}\right|} \lambda_{j i}^{\ell}\left(v_{2} \otimes \beta_{j}\right)\left(v_{1} \otimes \beta_{i}\right) \\
& =(-1)^{\left|v_{1}\right|\left|v_{2}\right|} T\left(v_{2}, v_{1}, \beta_{\ell}\right) .
\end{aligned}
$$

If $k>2$ and $m<k-1$, the result follows by induction. If $k>2$ and $m=k-1$, one can use Lemma 3.7 to reduce the result to the case $k=2$. We leave the details to the reader.

Now to the proof of Theorem 3.5. To begin with, a straightforward induction shows that $\rho$ is surjective. Indeed, any element $v_{1} \ldots v_{k} \otimes \beta_{\ell}$ can be expressed $\bmod I$ as a sum of products of terms of the form $v_{1} \ldots v_{m} \otimes \beta_{i}, m<k$. To prove $\rho$ injective, we define

$$
\sigma: Q\left[A \otimes B_{*}\right] \rightarrow Q\left[V \otimes B_{*}\right]
$$


by $\sigma\left(v_{1} \ldots v_{k} \otimes \beta_{\ell}\right)=(-1)^{\alpha\left(\left|\beta_{\ell}\right|\right)} T\left(v_{1}, \ldots, v_{k}, \beta_{\ell}\right)$. Then $\sigma$ is well defined by Lemma 3.8, $\sigma(I)=0$ by Lemma 3.7, and $\sigma \rho=$ identity by inspection. Thus $\rho$ is bijective and the theorem is proved.

\section{An Algebraic Model for $\Gamma(p)$}

In this section, we outline the modifications required to prove the analogues of the results of the previous two sections for the space of sections of a nilpotent fibration. We begin with an analogue of Theorem 1.2.

Suppose $p: E \rightarrow X$ is a fibration in $\Delta \mathcal{S}$ and let $\Gamma(p)$ be the simplicial form of the space of sections of $p$, that is,

$$
\Gamma(p)_{q}=\left\{f: \Delta[q] \times X \rightarrow E: p f=p_{2}\right\},
$$

where $p_{2}$ is the projection of $\Delta[q] \times X$ onto $X$. We say that $p: E \rightarrow X$ in $\Delta \mathcal{S}$ is nilpotent and of finite type if there is a sequence $E_{n} \rightarrow E_{n-1}$ of principal fibration with $E_{1}=X$ and with group and fibre $K\left(G_{n}, m_{n}\right)$ satisfying

(i) $G_{n}$ is a finitely generated abelian group, $m_{n} \leq m_{n+1}$, and $m_{n} \rightarrow \infty$ as $n \rightarrow \infty$.

(ii) There is an isomorphism $E \rightarrow \underset{\leftarrow}{\lim E_{n}}$ such that the diagram

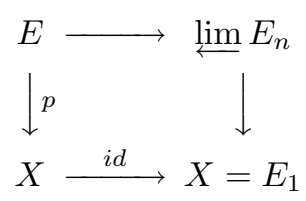

is commutative.

Let $B$ be a graded algebra and $V$ a graded vector space. We denote by $B[V]$ the graded algebra $B \otimes Q[V]$. A straightforward generalization of Theorem 2.6 of [BZ] yields the following:

Theorem 4.1. Let $p: E \rightarrow X$ be a nilpotent fibration as above, let $B \in \mathcal{A}$, and let $f: B \rightarrow \Omega(X)$ be a homology isomorphism. For each $n=1,2, \ldots$, let $V(n)$ be the graded vector space with

$$
\begin{aligned}
V(n)_{m_{n}} & =H o m\left(G_{n} ; Q\right), \\
V(n)_{j} & =0, \quad j \neq m_{n} .
\end{aligned}
$$

Set $V=\sum_{j=1}^{\infty} V(j)$ and $V[n]=\sum_{j=1}^{n} V(j)$. Then there is a differential d on $A=B[V]$ and a homology isomorphism $h: A \rightarrow \Omega(E)$ such that $d(V(n)) \subset B[V[n-1]]$, $d v$ is decomposable for $v \in V$ and the diagram

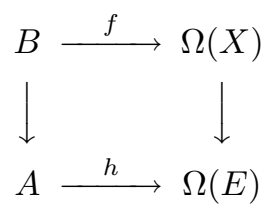

is commutative.

We next define an analogue of the space of sections in the category $\mathcal{A}$. Let $A=B[V]$ be as in Theorem 4.1 and $i: B \rightarrow A$ the inclusion. Then $\Gamma(i)$ is the 
simplicial set defined by

$$
\Gamma(i)=\{u \in \mathcal{F}(A, B): u(b)=1 \otimes b \text { for } b \in B\} .
$$

Recall that $\mathcal{F}(A, B) \in \Delta \mathcal{S}$ is the function space defined by

$$
\mathcal{F}(A, B)_{q}=\left(A, \Omega_{q} \otimes B\right)_{q} .
$$

The proof of Theorem 2.20 in [BZ] then carries over to produce

Theorem 4.2. If $i: B \rightarrow A$ is as above, then the components of $\Gamma(i)$ include the $Q$ - localizations of the components of $\Gamma(p)$.

We now identify $\Gamma(i)$ with a simplicial subset of $\Delta\left(Q\left(A \otimes B_{*}\right) / I\right) \approx \mathcal{F}(A, B)$. Recall that in Section 3 we defined an isomorphism $\Psi:\left(A \otimes B_{*}, \Omega_{q}\right) \rightarrow\left(A, \Omega_{q} \otimes B\right)$ given by

$$
\Psi(w)(a)=\sum_{i}(-1)^{\alpha\left(\left|b_{i}\right|\right)} w\left(a \otimes \beta_{i}\right) \otimes b_{i}
$$

We need to determine which $w$ satisfy $\Psi(w)(b)=1 \otimes b$, that is,

$$
\Psi(w)\left(b_{j}\right)=\sum_{i}(-1)^{\alpha\left(\left|b_{j}\right|\right)} w\left(b_{j} \otimes \beta_{i}\right) \otimes b_{i}=1 \otimes b_{j}
$$

or, equivalently,

$$
w\left(b_{j} \otimes \beta_{i}\right)-(-1)^{\alpha\left(\left|b_{j}\right|\right)} \beta_{i}\left(b_{j}\right)=0
$$

for all $i$ and $j$. Let $J \subset Q\left[A \otimes B_{*}\right]$ be the ideal generated by $I$ and all elements of the form $b \otimes \beta-(-1)^{\alpha(|b|)} \beta(b), b \in B, \beta \in B_{*}$.

Theorem 4.3. The mapping $\Psi$ induces an isomorphism

$$
\Delta\left(Q\left[A \otimes B_{*}\right] / J\right) \approx \Gamma(i) .
$$

We alter the proof of Theorem 3.5 slightly to prove

Theorem 4.4. The inclusion $V \subset B(V)=A$ induces an isomorphism

$$
Q\left[V \otimes B_{*}\right] \approx Q\left[A \otimes B_{*}\right] / J
$$

Proof. Note that $A \otimes B_{*}$ is spanned by elements of the form $b v_{1} v_{2} \cdots v_{\ell} \otimes \beta, b \in$ $B, v_{1}, \ldots, v_{\ell} \in V, \beta \in B_{*}$.

Define

$$
T\left(b, v_{1}, v_{2}, \ldots, v_{k}, \beta_{\ell}\right)=\sum \beta_{i j}^{\ell} T\left(v_{1}, v_{2}, \ldots, v_{k}, \beta_{j}\right),
$$

where $T\left(v_{1}, v_{2}, \ldots, v_{k}, \beta_{j}\right)$ is as in the proof of Theorem 3.5 and

$$
\beta_{i j}^{\ell}=\beta_{i}(b) \lambda_{i j}^{\ell}(-1)^{\varepsilon},
$$

where $\varepsilon=\alpha\left(\left|\beta_{i}\right|\right)+\left(\sum\left|v_{t}\right|\right)\left|\beta_{i}\right|$. The proof of Theorem 4.4 then proceeds exactly as in Theorem 3.5. 


\section{A Reduction of $Q\left[V \otimes B_{*}\right]$ то $Q\left[V \otimes H_{*}\left(B_{*}\right)\right]$}

Suppose $A=Q[V]$ and $B$ are as in Corolary 3.6. Let $\left\{v_{i}\right\}$ be a basis for $V$ such that $\left|v_{i}\right|>0,\left|v_{i}\right| \leq\left|v_{i+1}\right|$ and $d v_{i+1} \in Q\left[V_{i}\right]$, where $V_{i}$ is the subspace spanned by $v_{1}, \ldots, v_{i}$. Note that $d v_{i}$ is decomposable and $\left|v_{i}\right| \rightarrow \infty$ as $i \rightarrow \infty$. Let $\left\{a_{i}, b_{i}, c_{i}\right\}$ be a basis for $B_{*}$ with $d^{*} a_{i}=b_{i}$ and $d^{*} c_{i}=0$.

Lemma 5.1. The algebra $Q\left[V \otimes B_{*}\right]$ has a free set of generators $w_{i j}, u_{i j}$ and $v_{i j}$ satisfying

(i) $w_{i j}=v_{i} \otimes c_{j}+x_{i j}$, where $x_{i j} \in Q\left[V_{i-1} \otimes B_{*}\right]$,

(ii) $u_{i j}=v_{i} \otimes a_{j}$ and $v_{i j}=d u_{i j}$,

(iii) $d w_{i j} \in Q\left[\left\{w_{k \ell}: k<i\right\}\right]$,

(iv) if $d\left(v_{i} \otimes c_{j}\right)$ is decomposable, so is $d w_{i j}$.

Let $W$ be the subspace of $Q\left[V \otimes B_{*}\right]$ spanned by $\left\{w_{i j}\right\}$ and $U$ the subspace of $Q\left[V \otimes B_{*}\right]$ spanned by $\left\{u_{i j}, v_{i j}\right\}$. Thus $W$ is isomorphic to $V \otimes H_{*}\left(B_{*}\right)$ and $Q\left[V \otimes B_{*}\right]$ is isomorphic to $Q[W] \otimes Q[U]$.

Lemma 5.2. The algebra $Q[W]$ is a deformation retract (in the category $\mathcal{A}$ ) of $Q\left[V \otimes B_{*}\right]$.

(See $[\mathrm{BG}]$, Section 4 for a discussion of homotopy theory in the category $\mathcal{A}$.)

Before proving these lemmas, we state the following consequence.

Theorem 5.3. There is a differential on $Q\left[V \otimes H_{*}\left(B_{*}\right)\right]\left(\approx Q\left[w_{i j}\right]\right)$ making it an FNF (as in section 1) algebra such that

$$
Q\left[V \otimes B_{*}\right] \approx Q\left[V \otimes H_{*}\left(B_{*}\right)\right] \otimes Q\left[\left\{u_{i j}, v_{i j}\right\}\right]
$$

as DGA algebras (where $d u_{i j}=v_{i j}$ ). Furthermore $Q\left[V \otimes H_{*}\left(B_{*}\right)\right]$, with this differential, is a deformation retract of $Q\left[V \otimes B_{*}\right]$ in the category $\mathcal{A}$. Thus, $\Delta\left(Q\left[V \otimes B_{*}\right]\right)$ has the homotopy type of $\Delta\left(Q\left[V \otimes H_{*}\left(B_{*}\right)\right]\right)$.

Proof of Lemma 5.1. We use induction on $i$. Let $w_{1 j}=v_{1} \otimes c_{j}, u_{1 j}=v_{1} \otimes a_{j}$ and $v_{1 j}=d u_{1 j}$. Then $d w_{i j}=0$, since $d v_{1}=0$ and conditions (i),...,(iv) are satisfied. Suppose $w_{k, \ell}$ have been defined for $k<i$ and satisfy (i),...,(iv). Using induction and the fact that

$$
\begin{aligned}
v_{k \ell} & =d\left(v_{k} \otimes a_{\ell}\right) \\
& = \pm v_{k} \otimes b_{\ell}+\left(d v_{k}\right) \otimes a_{\ell} \\
& = \pm v_{k} \otimes b_{\ell} \quad \bmod Q\left[V_{k-1} \otimes B_{*}\right],
\end{aligned}
$$

we have $w_{k \ell}=v_{k} \otimes c_{\ell}, u_{k \ell}=v_{k} \otimes a_{\ell}$ and $v_{k, \ell}=v_{k} \otimes b_{\ell}$, modulo $Q\left[V_{k-1} \otimes B_{*}\right]$. Since $v_{k} \otimes c_{\ell}, v_{k} \otimes a_{\ell}$ and $v_{k} \otimes b_{\ell}, k<i$, freely generate $Q\left[V_{i-1} \otimes B_{*}\right]$, it follows that $w_{k, \ell}, u_{k, \ell}$ and $v_{k \ell}, k<i$, freely generate $Q\left[V_{i-1} \otimes B_{*}\right]$. Hence, we have

$$
Q\left[V_{i-1} \otimes B_{*}\right]=Q\left[w_{k \ell}: k<i\right] \otimes Q\left[u_{k \ell}, v_{k \ell}: k<i\right]
$$

as $D G$ algebras. Since

$$
d\left(v_{i} \otimes c_{j}\right)=\left(d v_{i}\right) \otimes c_{j} \in Q\left[V_{i-1} \otimes B_{*}\right]
$$

is a cycle,

$$
d\left(v_{i} \otimes c_{j}\right)=x+d u,
$$

where $x \in Q\left[w_{j k}: j<i\right]$ and $u \in Q\left[V_{i-1} \otimes B_{*}\right]$. Let $w_{i j}=v_{i} \otimes c_{j}-u$. Then $d w_{i j}=x$, as specified by 5.1 (iii). 
Suppose $d\left(v_{i} \otimes c_{j}\right)$ is decomposable and $d w_{i j}=x$ as above. Then

$$
\begin{aligned}
& x=\sum_{k<i} \delta_{k \ell} w_{k}, \\
& u=\sum_{k<i} \alpha_{k \ell} u_{k \ell}+\sum_{k<i} \beta_{k \ell} v_{k \ell}+\sum_{k<i} \gamma_{k \ell} w_{k \ell},
\end{aligned}
$$

mod decomposables. Since $d v_{k \ell}=0, d\left(\beta_{k \ell} v_{k \ell}\right)$ will not contribute to $d u$ and hence we may assume $\beta_{k \ell}=0$. Furthermore, the $d\left(\gamma_{k \ell} w_{k \ell}\right)$ terms could be shifted to the decomposable part $x$ in the formula

$$
d\left(v_{i} \otimes c_{j}\right)=x+d u,
$$

so that we can assume $\gamma_{k \ell}=0$. Hence

$$
d\left(v_{i} \otimes c_{j}\right)=\sum \delta_{k \ell} w_{k \ell}+\sum \alpha_{k \ell} v_{k \ell}
$$

mod decomposables. Therefore $\delta_{k \ell}=\alpha_{k \ell}=0$ and $d w_{i j}=x$ is decomposable.

Proof of Lemma 5.2. Define the $D G$ algebra mapping

$$
Q[W] \stackrel{j}{\rightarrow} Q[W] \otimes Q[u] \stackrel{r}{\rightarrow} Q[W]
$$

in the obvious way, and define a deformation retract

$$
H: Q[W] \otimes Q[U] \rightarrow \Omega_{1} \otimes Q[W] \otimes Q[U]
$$

by

$$
\begin{aligned}
H\left(w_{i j}\right) & =w_{i j}, \\
H\left(u_{i j}\right) & =u_{i j}(1-t), \\
H\left(v_{i j}\right) & =v_{i j}(1-t)+(-1)^{\left|v_{i j}\right|} u_{i j} d t .
\end{aligned}
$$

A straightforward computation shows that $H$ has the required properties.

We now prove Theorem 1.9. Since $B$ is formal, we can find a $D G$ algebra mapping $h: B \rightarrow H_{*}(B)$ extending the projection $Z_{*}(B) \rightarrow H_{*}(B)$. Then

$$
h^{*}: H_{*}(B)^{*}=H_{*}\left(B_{*}\right) \rightarrow B_{*}
$$

is a coalgebra map. Let $\alpha_{i}$ be a basis for $H_{*}\left(B_{*}\right)$. In choosing a basis $a_{i}, b_{i}, c_{i}$ as above, we may set $c_{i}=h^{*}\left(\alpha_{i}\right)$. If $D_{*}\left(\alpha_{i}\right)=\sum \varepsilon_{i}^{h \ell} \alpha_{\ell} \otimes \alpha_{j}$, then $D\left(c_{i}\right)=\sum \varepsilon_{i}^{h \ell} c_{h} \otimes c_{\ell}$. Thus $d\left(v_{i} \otimes c_{j}\right)$ will only involve terms consisting of products of $v_{j} \otimes c_{k}$, and hence, in the notation of the proof of Lemma 5.1, we may choose $w_{j k}=v_{j} \otimes c_{k}$. Then computing $d\left(v_{i} \otimes c_{j}\right)$ using $D$ will give the same result as computing $d\left(v_{i} \otimes \alpha_{j}\right)$ using $D_{*}$.

\section{Components of $\Delta(Q[W])$}

We now give an algebraic description of the components of $\Delta(Q[W])$, where $W$ is defined in the previous section.

Suppose $E=(Q[W], d), W \subset Q\left[V \otimes B_{*}\right]$, is as in Section 5 and let $\left\{x_{i}\right\}$ be a basis for $W$ satisfying $\left|x_{i}\right| \leq\left|x_{i+1}\right|$ and $d x_{i} \in Q\left[W_{i-1}\right]$, where $W_{i}$ is the subspace of $W$ spanned by the $x_{j}$ with $j<i$. Now, $E$ is a deformation retract of $Q\left[V \otimes B_{*}\right]$ (by Lemma 5.2), $\Delta Q\left[V \otimes B_{*}\right]$ is homotopy equivalent to $\mathcal{F}\left(X, Y_{Q}\right)$ (by Corollary 3.6), and $\mathcal{F}\left(X, Y_{Q}\right)$ is Kan when $Y_{Q}$ is Kan (see Theorem 6.9 of $[\mathrm{M}]$ ), which we are assuming. Thus $\Delta(E)$ is Kan, and it follows that the component of $\Delta(E)$ 
containing $u \in \Delta(E)_{0}$ is homotopy equivalent to $\Delta(E)_{u}$, the simplicial subset of $\Delta(E)$ consisting of those $w$ all of whose vertices are at $u$ :

$$
\Delta(E)_{u}=\left\{\omega \in \Delta(E) \mid \partial^{q-i} \partial^{i} \omega=u, \text { all } i, q=|w|\right\} .
$$

Let $K_{u}$ be the ideal in $E$ generated by the set

$$
\{w:|w|<0\} \cup\{d w:|w|=0\} \cup\{w-u(w):|w|=0\} .
$$

Theorem 6.1. The ideal $K_{u}$ is closed under differentation and the quotient map $E \rightarrow E / K_{u}$ induces a homotopy equivalence

$$
\Delta\left(E / K_{u}\right) \rightarrow \Delta(E)_{u} .
$$

Furthermore, $E / K_{u}$ is FNF, and is isomorphic to $Q[\tilde{W}]$, where $\tilde{W}^{q}=0$ for $q<$ $1, \tilde{W}^{1} \subset W^{1}$, and $\tilde{W}^{q}=W^{q}$ for $q>1$. Finally, if $u=0$ and $d w$ is decomposable for all $w \in W$, then $E / K_{u}$ is minimal and $\tilde{W}^{1}=W^{1}$.

Proof. Note first of all that if $w \in \Delta(E)$ vanishes on $K_{u}$, then, for $w \in W^{0}$, we have

$$
\omega(w)=\omega(w-u(w)+u(w))=u(w),
$$

since $u(w) \in Q$. Thus, $u$ is the only 0 -simplex in $\Delta\left(E / K_{u}\right)$, so that $\Delta\left(E / K_{u}\right) \subset$ $\Delta(E)_{u}$.

To prove that $d K_{u} \subset K_{u}$, it is enough to prove that $d \omega \in K u$ for $\omega$ in each of the three sets of generators defining $K_{u}$. If $w \in E^{-1}$, then

$$
d w=d w-u(d w)=\bar{w}-u(\bar{w}) \in K_{u},
$$

since $u(d w)=d u(w)=0$. We next prove $E / K_{u}=Q[\tilde{W}]$. Let $K=K_{u}$ and let $L_{n} \subset A$ be the ideal generated by the set

$$
\left\{x_{n}: m<n \text { and }\left|x_{n}\right|<0\right\} \cup\left\{x_{n}-u\left(x_{n}\right): m<n \text { and }\left|x_{n}\right|=0\right\} .
$$

Then, if $\left|x_{n}\right|=0$,

$$
d x_{n}=\sum_{\substack{\left|x_{m}\right|=1 \\ m<n}} a_{n m} x_{m} \bmod L_{n}
$$

$a_{n, m} \in Q$. Hence, we can change bases among the $x_{n}$ 's of dimension zero into one of the form

$$
\bar{x}_{n}=c_{n} x_{n}+\sum_{\substack{m<n \\\left|x_{m}\right|=1}} b_{n m} x_{m},
$$

where $c_{n}, b_{n, m} \in Q, c_{n} \neq 0$, such that if $\left|\bar{x}_{n}\right|=0, d \bar{x}_{n}=\bar{x}_{m}$ or $0 \bmod L_{n}$. Note that $\bar{x}_{m} \in W^{1}$ is in $K$ if and only if $\bar{x}_{m}=d \bar{x}_{n}$. Hence

$$
E / K=Q\left[x_{n}:\left|x_{n}\right|>1\right] \otimes Q\left[\bar{x}_{m}:\left|\bar{x}_{m}\right|=1, \bar{x}_{m} \neq d \bar{x}_{n}\right] .
$$

Furthermore, the differential of a generator in $E / K$ only involoves previous generators, so that $E / K$ is $\mathrm{FNF}$.

We next prove $E \rightarrow E / K$ induces a homotopy equivalence of $\Delta(E / K)$ into $\Delta(E)_{u}$. Let $E_{n}=Q\left[W_{n}\right]$. It is sufficient to prove that $E_{n} \rightarrow E_{n} / K$ induces a homotopy equivalence, since $\Delta(E) \rightarrow \Delta\left(E_{n}\right)$ is a fibration with fibre whose connectivity increases with $n$. We proceed by induction on $n$. The case $n=0$ is immediate. Suppose it is true for $n-1$. Let $u_{n}=u \mid \Delta\left(E_{n}\right)$ and let $K_{n}$ be the ideal $K$ for $E_{n}$. If $\left|x_{n}\right|<-1$, then $\Delta\left(E / K_{n}\right)=\Delta\left(E_{n-1} / K_{n-1}\right)$ and $\Delta\left(E_{n}\right)_{u_{n}}=$ 
$\Delta\left(E_{n-1}\right)_{u_{n-1}}$ by definition. If $\left|x_{n}\right|=-1$, then $\Delta\left(E_{n} / K_{n}\right)=\Delta\left(E_{n-1} / K_{n-1}\right)$ by definition. If $\omega \in \Delta\left(E_{n-1}\right)_{u_{n-1}}$ and $|w|=q$, then $d \omega\left(d x_{n}\right)=0$ and $\partial_{0}^{q} w\left(d x_{n}\right)=$ $u\left(d x_{n}\right)=0$, so that $w\left(d x_{n}\right)=0$. We then have

$$
\begin{aligned}
\Delta\left(E_{n-1}\right)_{u_{n-1}} & =\left\{\omega \in \Delta\left(E_{n-1}\right)_{u_{n-1}}: \omega\left(d x_{n}\right)=0\right\} \\
& =\Delta\left(E_{n}\right)_{u_{n}} .
\end{aligned}
$$

Suppose $\left|x_{n}\right| \geq 0$. We then have fibrations $p_{1}$ and $p_{2}$ in the following commutative diagram with $\Delta Q\left[x_{n}\right]$ the fibre of both:

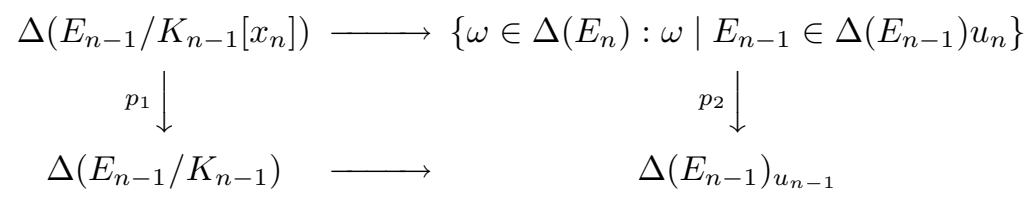

For $\left|x_{n}\right|>0$ this gives a homotopy equivalence $\Delta\left(E_{n} / K_{n}\right) \rightarrow \Delta\left(E_{n}\right)_{u_{n}}$. If $\left|x_{n}\right|=0$, then $p_{1}$ and $p_{2}$ are covering projections. Let $v \in \Delta\left(\left(E_{n-1} / K_{n-1}\right)\left[x_{n}\right]\right)_{0}$ be defined by $v\left(x_{n}\right)=u\left(x_{n}\right)$. Then $v$ maps to $u_{n}$ above and, passing to components, we have a homotopy equivalence

$$
\Delta\left(E_{n-1} / K_{n-1}\left[x_{n}\right]\right)_{v} \rightarrow \Delta\left(E_{n}\right)_{u_{n}} .
$$

Thus it is sufficient to show that

$$
\Delta\left(E_{n} / K_{n}\right) \rightarrow \Delta\left(E_{n-1} / K_{n-1}\left[x_{n}\right]\right)_{v}
$$

is a homotopy equivalence. If $d x_{n} \in K_{n-1}$, the two sides are equal, since $d \omega\left(x_{n}\right)=0$ and $\partial_{0}^{q} \omega=u_{n}$ implies $\omega\left(x_{n}\right)=u\left(x_{n}\right)$. Suppose $d x_{n} \notin K_{n-1}$. Then, as in the proof of freeness above, we may assume $d x_{n}=x_{m}, m<n$. Let $x=x_{n}$ and $y=x_{m}$. If $j<i$, then $d x_{j}=\alpha_{j}+y \beta_{j}$ in $E_{n-1} / K_{n-1}$ and $\alpha_{j}, \beta_{j} \in E_{j-1}$. Let

$$
\bar{x}_{j}=x_{j}-x \beta_{j}, \quad j \geq 1, j \neq m .
$$

One can solve for the $x_{i}$ 's in terms of $\bar{x}_{j}$ 's, and hence $d \bar{x}_{j} \in Q\left[\bar{x}_{j}: i<j\right]$. Thus

$$
E_{n-1} / K_{n-1}\left[x_{n}\right]=Q\left[\bar{x}_{j}\right] \otimes Q[x, y],
$$

as $D G$ algebras. Hence $Q\left[\bar{x}_{j}\right]$ is a deformation retract of $E_{n-1} / K_{n-1}\left[x_{n}\right]$ as in Lemma 5.1. The image of $\bar{x}_{j}$ under the map

$$
Q\left[\bar{x}_{j}\right] \rightarrow E_{n-1} / K_{n-1}\left[x_{n}\right] \rightarrow E_{n} / K_{n}
$$

is $x_{j}-u\left(x_{0}\right) \beta_{j}$. Since $\beta_{j}$ is a polynomial in $x_{k}, k<j$, this map is an isomorphism. Hence

$$
\Delta\left(E_{n} / K_{n}\right) \rightarrow \Delta\left(E_{n-1} / K_{n-1}\left[x_{n}\right]\right)_{v} \rightarrow \Delta\left(Q\left[\bar{x}_{j}\right]\right)
$$

are homotopy equivalences.

Corollary 6.2. If $E=(Q[W], d)$ is as in Theorem 6.1 and $u \in \Delta(E)_{0}$, there are $\bar{W}$ and $\bar{d}$ such that $\bar{W}$ is isomorphic to a quotient of $W, \bar{E}=(Q[\bar{W}], \bar{d})$ is minimal and $\Delta(\bar{E})$ is homotopy equivalent to $\Delta(E)_{u}$.

Proof. In [BG], Propositions 7.11 shows that such an $\bar{E}$ exists which is homotopy equivalent to $\tilde{E}$ as in Lemma 5.2 . 


\section{Two Examples}

In this section we present two examples. In the first, we determine the set of components and describe a minimal model for each component. It follows from this description that not all components have the same homotopy type. The second example shows that the formality condition is necessary in Theorem 1.9.

Let $A=Q[V]$, where $V$ has a basis $\{x, y, v\}$ with

$$
\begin{aligned}
& |x|=2, d x=0, \\
& |y|=4, d y=0, \\
& |v|=5, d v=x y .
\end{aligned}
$$

Let $B_{*}=Q[\alpha, \beta]$ be the Hopf algebra with $\alpha, \beta$ primitive and with $|\alpha|=-2,|\beta|=$ -3 , and $d \alpha=d \beta=0$. Let $W=V \otimes B_{*}$ and let $Q[W]$ be the $D G$ algebra with differential induced by the differential in $Q\left[A \otimes B_{*}\right]$ as in Corollary 3.6. Then $Q[W]$ in dimension $q=-1,0,1,2$ has a basis as follows (we write $x \alpha$ for $x \otimes \alpha$ and $x$ for $x \otimes 1):$

$$
\begin{aligned}
& \text { degree }-1: x \beta, y \alpha \beta, v \alpha^{3}, \\
& \text { degree } 0: x \alpha, y \alpha^{2}, v \alpha \beta, \\
& \text { degree } 1: y \beta, v \alpha^{2}, \\
& \text { degree } 2: x, y \alpha, v \beta .
\end{aligned}
$$

Ignoring terms involving negative dimensional generators, the non-zero differentials of the basis above are given by

$$
\begin{aligned}
d\left(v \alpha^{3}\right) & =3(x \alpha)\left(y \alpha^{2}\right), \\
d(v \alpha \beta) & =(x \alpha)(y \beta), \\
d\left(v \alpha^{2}\right) & =2(x \alpha)(y \alpha)+x\left(y \alpha^{2}\right), \\
d(v \beta) & =x(y \beta) .
\end{aligned}
$$

For example,

$$
\begin{aligned}
d(v \alpha \beta) & =d(v \otimes \alpha \beta)=x y \otimes \alpha \beta \\
& =(x \otimes 1)(y \otimes \alpha \beta)+(x \otimes \alpha \beta)(y \otimes 1)+(x \otimes \alpha)(y \otimes \beta)+(x \otimes \beta)(y \otimes \alpha),
\end{aligned}
$$

using equation (1.1), since

$$
\begin{aligned}
D(\alpha \beta) & =D(\alpha) D(\beta) \\
& =(1 \otimes \alpha+\alpha \otimes 1)(1 \otimes \beta+\beta \otimes 1) .
\end{aligned}
$$

However, the first, second, and fourth terms involve negative dimensional generators so that

$$
\begin{aligned}
d(v \alpha \beta) & =(x \otimes \alpha)(y \otimes \beta) \\
& =(x \alpha)(y \beta) .
\end{aligned}
$$

The other cases are similar.

We now compute the set of components of $\Delta(Q[W])$. Note first of all that the set $\Delta(Q[W])_{0}$ of 0 -simplices can be identified with the set of mappings $u: W^{0} \rightarrow Q$ satisfying $u(d w)=0$ for any $w \in W^{-1}$. Thus, $u$ is determined by $a, b, c \in Q$, where

$$
u(x \alpha)=a, u\left(y \alpha^{2}\right)=b, u(v \alpha \beta)=c .
$$


The condition $u(d w)=0$ implies that $a b=0$, so that

$$
\Delta(Q[W])_{0} \approx\left\{(a, b, c) \in Q^{3}: a b=0\right\} .
$$

Let $u_{a, b, c}$ denote the element of $\Delta(Q[W])_{0}$ corresponding to $(a, b, c)$.

In the same way, it can be shown that the set $\Delta(Q[W])_{1}$ of 1 -simplices of $\Delta(Q[W])$ consists of all pairs $(u, s)$, where

$$
u: W^{0} \rightarrow Q[t], \quad s: W^{1} \rightarrow Q[t, d t]
$$

satisfy $u(d w)=0$ for $w \in W^{-1}$ and $s(d z)=d u(z)$ for $z \in W^{0}$. Suppose that

$$
\begin{aligned}
u(x \alpha) & =a(t), \\
u\left(y \alpha^{2}\right) & =b(t), \\
u(v \alpha \beta) & =c(t), \\
s(y \beta) & =f(t) d t, \\
s\left(v \alpha^{2}\right) & =g(t) d t .
\end{aligned}
$$

Then,

$$
0=u(d(x \alpha))=d u(x \alpha)=\alpha^{\prime}(t) d t
$$

so $a(t)=a$ is a constant. Similarly, $b(t)=b$ is a constant, $a b=0$, and $c^{\prime}(t)=a f(t)$. Thus $\Delta(Q[W])_{1}$ can be identified with the set of 5 -tuples $(a, b, c(t), f(t), g(t))$, where $a, b \in Q, c(t), f(t), g(t) \in Q[t]$. Note that, with this identification, $\partial_{0}(u, s)$ is the zero simplex corresponding to $(a, b, c(0))$ and $\partial_{1}(u, s)$ is the zero simplex corresponding to $(a, b, c(1))$.

Suppose that $(u, s)$ is as above and $a=0$. Then $c^{\prime}(t)=0$ and $c(t)=c$ is constant. It follows that $\partial_{0}(u, s)=\partial_{1}(u, s)$, so that the component of $\Delta(Q[W])$ containing the zero simplex $u_{0, b, c}$ contains no other 0 -simplices. Thus, each $u_{0, b, c}$ determines a distinct component.

Next, suppose that $a \neq 0$ and $b=0$ and that $u_{a, 0,0}, u_{a, 0, c} \in \Delta(Q[W])_{0}$. Let $(u, s) \in \Delta(Q[W])_{1}$ correspond to $\left(a, 0, c(t), c^{\prime}(t) / a, 0\right)$, where $c(0)=0$ and $c(1)=c$. Then $\partial_{0}(u, s)=w_{a, 0,0}$ and $\partial_{1}(u, s)=w_{a, 0, c}$, so that each 0 -simplex $w_{a, 0, c}$ lies in the same component as $w_{a, 0,0}$.

It follows from the discussion above that $\pi_{0}(\Delta(Q[W]))$, the set of components of $\Delta(Q[W])$, is given by

$$
\pi_{0}(\Delta(Q[W])) \approx Q^{2} \cup\{Q-(0)\}
$$

Let $u=u_{0,0,0}, w=u_{1,0,0} \in \Delta(Q[W])_{0}$. A straightforward computation shows that the ideal $K_{u}$ (defined in Section 6) is generated by all $z \in Q[W]$ with $|z|<0$ together with the elements $x \alpha, y \alpha^{2}, v \alpha \beta$. Similarly, the ideal $K_{w}$ is generated by all $z \in Q[W]$ with $|z|<0$ together with $x \alpha-1, y \alpha^{2}, v \alpha \beta, y \beta$. Set $A_{u}=Q[W] / K_{u}$ and $A_{w}=Q[W] / K_{w}$. According to Theorem 6.1, the component of $\Delta(Q[W])$ containing $u$ is homotopy equivalent to $\Delta\left(A_{u}\right)$ and the component of $\Delta(Q[W])$ containing $w$ is homotopy equivalent to $\Delta\left(A_{w}\right)$. Now, $A_{u}^{1}$ has basis $y \beta, v \alpha^{2}$ with $d(y \beta)=d\left(v \alpha^{2}\right)=0$ and $A_{w}^{1}$ has basis $v \alpha^{2}$ with $d\left(v \alpha^{2}\right)=2 y \alpha$. It follows that

$$
\begin{aligned}
& H^{1}\left(\Delta(Q[W])_{u}\right) \simeq Q^{2}, \\
& H^{1}\left(\Delta(Q[W])_{w}\right) \simeq 0,
\end{aligned}
$$

so that the components $\Delta(Q[W])_{u}$ and $\Delta(Q[W])_{w}$ have different homotopy types. 
We note that $A_{u}$ is a minimal model for $\Delta(Q[W])_{u}$. Applying Theorem 6.1, we see that the subalgebra of $A_{w}$ generated by $x, v \beta, v-x(v \alpha)+(x)^{2}\left(v \alpha^{2}\right)$ is a minimal model for $\Delta(Q[W])_{w}$.

We next calculate differentials in a nonformal example. This yields a result different from what is obtained using the coproduct in $H_{*}\left(B_{*}\right)$ as in the formal case.

Let $A=Q[V]$, where $V$ has a basis $\left\{v_{1}, v_{2}, v_{3}, v_{4}\right\}$ with

$$
\begin{aligned}
& \left|v_{1}\right|=3, d v_{1}=0, \\
& \left|v_{2}\right|=2, d v_{2}=0, \\
& \left|v_{3}\right|=4, d v_{3}=v_{1} v_{2}, \\
& \left|v_{4}\right|=6, d v_{4}=v_{1} v_{3} .
\end{aligned}
$$

Let $B=Q[x, y, z, u, w]$, where $U$ has a basis $\{x, y, z, u, w\}$ with

$$
\begin{aligned}
& |x|=|y|=|z|=2, d x=d y=d z=0, \\
& |u|=|w|=3, d u=x y, \quad d v=y z,
\end{aligned}
$$

and consider the sets

$$
\begin{aligned}
& \gamma=\left\{x, y, z, x^{2}, y^{2}, z^{2}, x z, \sigma=u z-x w\right\}, \\
& \alpha=\{u, v, x u, y u, x w, y w, z w\}, \\
& \beta=\{x y, y z\} .
\end{aligned}
$$

Then $\alpha \cup \beta \cup \gamma$ is a basis for $B^{1} \oplus \cdots \oplus B^{5}$ and we let $\alpha^{*} \cup \beta^{*} \cup \gamma^{*}$ be the dual basis for $\left(B^{1} \oplus \cdots \oplus B^{5}\right)^{*}$. The basis $\alpha^{*} \cup \beta^{*} \cup \gamma^{*}$ is of the kind required in Section 5. More explicitly, the elements of $\gamma^{*}$ correspond to the $c_{i}$, the elements of $\alpha^{*}$ correspond to the $a_{i}$, and the elements of $\beta^{*}$ correspond to the $b_{i}$.

We now construct the elements $w_{i j} \in Q\left[V \otimes B_{*}\right]$ satisfying (i) and (iii) of Theorem 5.1. Let $w_{i j}=v_{i} \otimes c_{j}, i, j=1, \ldots, 4, c_{j} \in \gamma^{*}$ except for $c_{j}=\sigma^{*}, i=3,4$. Then (i) and (iii) of Theorem 5.1 are satisfied for the $w_{i j}$ and we need only define

$$
\begin{array}{ll}
w_{1}=v_{3} \otimes \sigma^{*} & \bmod Q\left[V_{2} \otimes B_{*}\right], \\
w_{2}=v_{4} \otimes \sigma^{*} & \bmod Q\left[V_{3} \otimes B_{*}\right],
\end{array}
$$

with $d w_{1}$ and $d w_{2}$ satisfying (iii). A straightforward computation shows that

$$
D \sigma^{*}=1 \otimes \sigma^{*}+\sigma^{*} \otimes 1+u^{*} \otimes z^{*}+z^{*} \otimes u^{*},
$$

so that

$$
\begin{aligned}
d\left(v_{3} \otimes \sigma^{*}\right)= & v_{1} v_{2} \otimes \sigma^{*} \\
= & \left(\alpha_{1} \otimes \sigma^{*}\right)\left(v_{2} \otimes 1^{*}\right)+\left(v_{1} \otimes 1^{*}\right)\left(v_{2} \otimes \sigma^{*}\right) \\
& \quad+\left(v_{1} \otimes z^{*}\right)\left(v_{2} \otimes u^{*}\right)+\left(v_{1} \otimes u^{*}\right)\left(v_{2} \otimes z^{*}\right) .
\end{aligned}
$$

The first two terms in this expression are of the required form but the last two are not. To eliminate the last two terms, we note that

$$
\begin{aligned}
d\left(\left(v_{2} \otimes z^{*}\right)\left(v_{2} \otimes(x y)^{*}\right)\right. & \left.+\left(v_{1} \otimes(x y)^{*}\right)\left(v_{2} \otimes z^{*}\right)\right) \\
& =-\left(v_{1} \otimes z^{*}\right)\left(v_{2} \otimes u^{*}\right)-\left(v_{1} \otimes u^{*}\right)\left(v_{2} \otimes z^{*}\right) .
\end{aligned}
$$

Defining $w_{1}$ by

$$
w_{1}=v_{3} \otimes \sigma^{*}+\left(v_{1} \otimes z^{*}\right)\left(v_{2} \otimes x y^{*}\right)+\left(v_{1} \otimes x y^{*}\right)\left(v_{2} \otimes z^{*}\right),
$$

we see that $d w_{1}$ has the required form. 
In the same way, we define $w_{2}$ by

$$
w_{2}=v_{4} \otimes \sigma^{*}+\left(v_{1} \otimes z^{*}\right)\left(v_{3} \otimes x y^{*}\right)+\left(v_{1} \otimes x y^{*}\right)\left(v_{3} \otimes z^{*}\right) .
$$

Then

$$
\begin{aligned}
d w_{2}= & \left(v_{1} \otimes \sigma^{*}\right)\left(v_{3} \otimes 1^{*}\right)+\left(v_{1} \otimes 1^{*}\right)\left(w_{1}\right) \\
& -\left(v_{1} \otimes z^{*}\right)\left(v_{1} \otimes x^{*}\right)\left(v_{2} \otimes y^{*}\right) \\
& -\left(v_{1} \otimes z^{*}\right)\left(v_{1} \otimes y^{*}\right)\left(v_{2} \otimes x^{*}\right),
\end{aligned}
$$

which has the required form.

On the other hand, if $\tilde{\sigma}$ is the element of $H_{*}\left(B_{*}\right)$ determined by $\sigma^{*}$, then

$$
\tilde{D} \tilde{\sigma}=\tilde{\sigma} \otimes 1+1 \otimes \tilde{\sigma},
$$

where $\tilde{D}$ is the coproduct in $H_{*}\left(B_{*}\right)$. We then have

$$
d\left(v_{4} \otimes \tilde{\sigma}\right)=v_{1} v_{3} \otimes \tilde{\sigma}=\left(v_{1} \otimes \tilde{\sigma}\right)\left(v_{3} \otimes 1\right)+\left(v_{1} \otimes 1\right)\left(v_{3} \otimes \tilde{\sigma}\right) .
$$

\section{BIBLIOGRAPHY}

[BG] A.K. Bousfield and V.K.A.M. Gugenheim, On PL de Rham theory and rational homotopy type, Mem. Amer. Math. Soc. No. 179 (1976). MR 54:13906

[BPS] A. Bousfield, C. Peterson, and L. Smith, The rational homology of function spaces, Arch. Math 52 (1989), 275-283. MR 90d:55020

[BZ] E.H. Brown and R.H. Szczarba, Continuous cohomology and real homotopy type, Trans. AMS 311 (1989), 57-106. MR 89f:55005

$[\mathrm{H}] \quad$ A. Haefliger, Rational homotopy of the space of sections of a nilpotent bundle, Trans. AMS 273 (1982), 609-620. MR 84a:55010

[Hi] P. Hilton, G. Mislin, and J. Roitberg, Localization of nilpotent groups and spaces, North Holland Mathematics Studies 15, North Holland, New York, 1975. MR 57:17635

[L] J. Lannes, Sur la cohomologie modulo $p$ des p-groupes abéliens élémentaires, Proc. Durham Symp. on Homotopy Theory 1985, LMS Lecture Note Ser., no. 117, Camb. Univ. Press, 1987, 97-116. MR 89e:55037

[M] J.P. May, Simplicial objects in algebraic topology, Univ. Chicago Press, Chicago IL., 1992. MR 93m:55025

[S] D. Sullivan, Infinitesimal computations in topology, Inst. Hautes Etudes Sci. Publ. Math. 47 (1977), 269-331. MR 58:31119

[VS] M. Vigué-Poirrier and D. Sullivan, The homology theory of the closed geodesic problem, J. Differential Geometry 11 (1976), 633-644. MR 56:13269

[V1] M. Vigué-Poirrier, Cohomologie de l'espace des sections d'un fibre et cohomologie de Gelfand-Fuchs d'une variete, Lecture Notes in Math., 1183, Springer-Verlag, 1986, 371396. MR 87i:55026

[V2] _ Sur l'homotopie rationnelle des espaces functionnels, Manuscripta Math. 56 (1986), 177-191. MR 87h:55009

[W] G.W. Whitehead, On products in homotopy groups, Ann. Math. 47 (1946), 460-475. MR 8:50b

Department of Mathematics, Brandeis University, Waltham, Massachusetts 02254

Department of Mathematics, Yale University, Box 208283, New Haven, Connecticut 06520 